\title{
NON VALVULAR ATRIAL FIBRILLATION AND ACUTE CORONARY SYNDROME- PRESENT INDIAN PERSPECTIVE AND ASSESSEMENT
}

\author{
Dr Ranjan Modi $^{1}$, Dr Aparna jaswal ${ }^{2}$, and Dr sunil modi ${ }^{1}$ \\ ${ }^{1}$ Indraprastha apollo Hospitals \\ ${ }^{2}$ Fortis Escorts heart Institute
}

August 14, 2020

\begin{abstract}
The number of patients undergoing percutaneous coronary intervention (PCI) who mandate additional oral anticoagulant therapy has been increasing. Dual antiplatelet therapy (DAPT) is associated with reduced ischemic events including stent thrombosis, myocardial infarction and stroke following PCI. However, the trade-off is an increased risk for bleeding while on DAPT. The addition of an anticoagulation further increases the likelihood of bleeding while on antiplatelet therapy. Thus, the overall risks and benefits for each patient undergoing PCI on anticoagulation (VKA/ NOAC) must be assessed and therapy individualized to ensure optimal therapy for each unique situation. We review the best practices for the pharmacologic management of patients requiring anticoagulation with NOAC who are treated with PCI and require antiplatelet therapy.
\end{abstract}

\section{Hosted file}

TABLE 3.docx available at https://authorea.com/users/350595/articles/475401-non-valvularatrial-fibrillation-and-acute-coronary-syndrome-present-indian-perspective-andassessement

\section{Hosted file}

TABLE 2.docx available at https://authorea.com/users/350595/articles/475401-non-valvularatrial-fibrillation-and-acute-coronary-syndrome-present-indian-perspective-andassessement

\section{Hosted file}

TABLE 1.docx available at https://authorea.com/users/350595/articles/475401-non-valvularatrial-fibrillation-and-acute-coronary-syndrome-present-indian-perspective-andassessement

Keywords: Antiplatelet therapy; Oral anticoagulant; Non-vitamin K antagonist oral anticoagulants; Percutaneous coronary intervention

\section{INTRODUCTION}

\section{Hosted file}


TITLE PAGE .docx available at https://authorea.com/users/350595/articles/475401-nonvalvular-atrial-fibrillation-and-acute-coronary-syndrome-present-indian-perspectiveand-assessement

Long-term oral anticoagulation $(\mathrm{OAC})$ is an essential aspect in the treatment of patients with atrial fibrillation (AF). Approximately 70-80\% of all patients in AF have an indication for lifelong OAC, and coronary artery disease coexists in 20-30\% of these patients. ${ }^{1,2}$ Balancing the risk of bleeding and thromboembolism is crucial in the management of patients on $\mathrm{OAC}$, even more in cases when $\mathrm{AF}$ is associated with percutaneous coronary intervention (PCI).

Furthermore as the number of available oral antiplatelet and oral anticoagulant agents continue to grow, so does the uncertainty about various permutations and combinations regarding optimal combination therapy. Given the high proportion of patients undergoing PCI on antiplatelets and requiring OAC for conditions particularly like AF, it is important that physicians are aware of the clinical implications and management of these overlapping conditions. The periprocedural management of anticoagulated patients is critical, but clinical practice varies widely between clinicians, hospitals, and countries. The addition of these oral anticoagulation often requires modification of the antiplatelet regimen to optimize patient outcomes by balancing the risk of bleeding with the risk for ischemic events.

The need of this study was to determine the diversity in the antiplatelet and anticoagulation treatment of patients with Non Valvular AF or flutter who develop ACS or undergo PCI in India. Thus, in view of the lack of data available, also, determining the guidance towards management of such patients in India.

\section{MATERIAL AND METHODS}

All patients who had AF and underwent PCI were included in the study.

\section{INCLUSION CRITERIA}

- Adults > 18 years of age (Male or Female).

- Active or history of NVAF or flutter on anticoagulation.

- Percutaneous coronary intervention with stenting (DES)

\section{RESULTS :}

In our study the majority of the subjects were males with the male to female ratio of 1.6:1.The mean age was $61 \pm 4.2$ years. The risk factors included diabetes (65\%), hypertension (58\%), dyslipidaemia( $57 \%)$ and smoking $(32 \%)$. The most common cardiac presentation in the patients was unstable angina 61patients followed by Non ST elevation MI (NSTEMI) 25 patients, ST elevation MI(STEMI) 8 patients and stable angina in 6 patients. Echocardiography revealed normal Left Ventricular (LV) function in 47 patients, mild LV dysfunction in 31 patients, moderate in 12 patients and severe LV dysfunction in 10 patients.

The mean CHA2DS2VASc score was 2.46 \pm 1.32 . Patients presenting with persistent AF were 53 patients, paroxysmal AF 35 patients and permanent AF in 12 patients. The patient were treated for single vessel disease in 51 cases, double vessel in 38 cases and triple vessel disease was noted in 11 patients.

The patients were started on triple therapy ( dual antiplatelet and anticoagulant ) in $95 \%$ cases with DAPT only seen in $5 \%$ cases. Amongst the anticoagulant therapy most of the patients were initiated on NOACs $85 \%$ with a few on $\operatorname{VKA}($ Acitrom) $15 \%$. None of the patients were started on warfarin. The bleeding manifestation were measured using BARC definitions which showed majority of the cases 78 in BARC 0 , 14 in BARC 1,6 in BARC 2 and 2 in BARC 3. No cases were reported in BARC 4 and 5 group.

A number of patients who presented with STEMI (8 patients), unstable angina (10 patients), were initially loaded with ticagrelor but with the advent of AF post loading dose, they were later shifted to clopidogrel the next day in view of the initiation of anticoagulation as well. Only 1 patient was loaded with prasugrel outside which was shifted to clopidogrel as per the same protocol. 
The patients were followed up at 1 month , 3 months, 6 months and one year for adjustment of antiplatelet, anticoagulation medicines and their dosage.

The study analyzed the evolving dilemma of peri-procedural and post-procedural management of anticoagulated patient's, burden of the disease, available data, risk factors that could identify high risk patients and propose a best well-balanced management strategy of antiplatelets and anticoagulation in patients with PCI and $\mathrm{AF}$.

In our study of patients with AF and ACS, we came across these scenarios. The management of the patients with antiplatelets, anticoagulants and their dosage differed in the groups with changes at initiation and follow up.

1. Recent ACS ( STEMI) with recent AF

2. Recent ACS ( NSTEMI) with recent AF

3. Recent ACS ( STEMI/ NSTEMI) with old AF on anticoagulation

\section{DISCUSSION ;}

$\mathrm{AF}$ is associated with a small but significant incidence of stroke and systemic thromboembolism. ${ }^{3}$ Oral anticoagulants (NOACs) reduce the incidence of stroke and systemic embolism in these patients. ${ }^{4} \mathrm{~A}$ metaanalysis of 29 trials on use of antithrombotic therapy to prevent stroke in patients who have nonvalvular atrial fibrillation. showed that anticogulation reduced stroke by $64 \%$ as compared with placebo and by $39 \%$ as compared with aspirin in patients with non-valvular $\mathrm{AF}^{5}$.

Furthermore several trials including ACTIVE-W have confirmed the superiority of anticoagulation in reducing embolic events over dual antiplatelet therapy (DAPT) with aspirin and clopidogrel in patients with both paroxysmal and sustained AF and at least 1 additional stroke risk factor ${ }^{6}$ As a result, the ACC/AHA guidelines recommended oral anticoagulant therapy for those patients with at least 1 additional risk factor for stroke and suggested the use of aspirin only for those at low risk for stroke such as patients without risk factors. ${ }^{3}$

Three antithrombotic drug combinations have been used most in practice in patients with AF and PCI: triple therapy (oral anticoagulation and dual antiplatelet therapy with aspirin and clopidogrel), oral anticoagulation, and 1 antiplatelet agent (aspirin or clopidogrel), or rarely, DAPT alone without oral anticoagulants. Although there are wide variations in type and duration of therapy in practice, initially triple therapy is the most common treatment regimen in this setting. Antiplatelets other than clopidogrel like prasugrel and ticagrelor have been sparingly used in combination with anticoagulants due to increased chance of bleeding events.

A study of 21,443 elderly patients on bleeding complications associated with combinations of aspirin, thienopyridine derivatives, and warfarin in elderly patients following acute myocardial infarction, when followed on average for 22 months after an acute MI, bleeding was 1.7 times more frequent with DAPT and 1.9 times more frequent with aspirin plus warfarin when compared with aspirin monotherapy. ${ }^{7}$ Similarly, in a nationwide registry of 40,812 patients with acute MI in Denmark, the risk of bleeding was $2.6 \%$ for aspirin, $4.6 \%$ for clopidogrel, $4.3 \%$ for DAPT, $5.1 \%$ for aspirin plus an oral anticoagulant, $12.3 \%$ for clopidogrel plus an oral anticoagulant, and $12.0 \%$ for triple therapy over a mean follow-up of 16 months ${ }^{8}$.

Thus the literature suggests that taking combination of oral anticoagulants with aspirin and clopidogrel ("triple therapy") pose a significant dilemma for the cardiologist because of the increased risk of major bleeding. Though the bleeding risk is higher as noted in various studies still triple therapy (TT) was the commonest regimen used in the setting of atrial fibrillation patients requiring PCI (Data from CRUSADE Registry). ${ }^{9}$ Similarly data from Society for Cardiac Angiography and Interventions survey (2011) revealed that $86 \%$ of interventionists prefer Triple Therapy for 1 month followed by warfarin and aspirin in case of bare-metal stent and $47.4 \%$ recommend at least 6 months of Triple Therapy after DES implantation.

According to guidelines DAPT alone is inferior to warfarin for prevention of thromboembolic events in 
patients with atrial fibrillation. ${ }^{10}$ Various trials addressed this issue of dual Therapy (Warfarin+ Clopidogrel) Vs. Triple Therapy. The results from the single center WOEST trial involving 573 patients who underwent PCI with a mean follow up of 1 year clearly show that use of dual therapy (Warfarin and clopidogrel without aspirin) was associated with a significant reduction in bleeding complications with no increase in the rate of thrombotic events were observed ${ }^{11}$.

Furthermore use of triple therapy was associated with higher mortality and morbidity ${ }^{12}$. Although data on the efficiency and safety of warfarin plus clopidogrel combination are limited, but this combination may be an alternative in patients with high bleeding risk and/or absent risk factors for stent thrombosis. The study did conclude that anticoagulants and clopidogrel was associated with significant reduction in major bleeding and no increase in thrombotic events compared to triple therapy with anticoagulation, aspirin and clopidogrel. ${ }^{12}$

In the past these patients received a (TT) for 3-12 months. This TT has never been studied for efficacy; however, the rate of bleeding complications in comparison to a simple OAC or DAPT is significantly higher. Registries and smaller trials showed that DAPT with an OAC plus a platelet inhibitor may be sufficient to prevent stroke and stent thromboses/myocardial infarctions.

In the past anticoagulation was limited to vitamin $\mathrm{K}$ antagonists (VKA), but with the recent addition of $\mathrm{NOAC}$ in the treatment regimen of $\mathrm{AF}$, the combination of antiplatelets and anticoagulants has become more wide range.

These questions were investigated in various prospective and randomized studies involving all four nonvitamin $\mathrm{K}$ oral anticoagulants (NOAC) approved for stroke prevention in AF. The NOACs were tested against vitamin $\mathrm{K}$ antagonists (VKA) involving single antiplatelet therapy without using DAPT. The trials with rivaroxaban (PIONEER AF-PCI), dabigatran (RE-DUAL PCI), apixaban (AUGUSTUS) and edoxaban (ENTRUST-AF PCI) are instrumental in determining the management. The current status is that a NOAC plus a single antiplatelet agent, mostly clopidogrel, is superior to TT with VKA with respect to bleeding complications without any obvious disadvantage due to increases in stroke cases or cardiac ischemia. The international guidelines already permit treatment without TT in cases where the bleeding risk is prevalent. In this situation it is recommended to prescribe a NOAC plus a single antiplatelet therapy. Thus, TT no longer seems to be indicated for most patients with AF and after ACS or PCI with exception of patients in high risk category like left main PCI, multivessel stenting PCI, Diabetes and multiple stents.

\section{NOVEL ORAL ANTICOAGULANTS}

NOAC has become widely adopted since initial approval. They represent a class of drugs that have two unique mechanisms of action; both directly inhibit a single coagulation factor with the distinction between individual types of agents occurring based on the coagulation factor that is inhibited. Apixaban, edoxaban and rivaroxaban each inhibit factor Xa, whereas the direct thrombin inhibitor dabigatran inhibits factor IIa (thrombin).

The introduction and rise of NOAC were in response to numerous limitations and challenges with the preexisting therapy of vitamin $\mathrm{K}$ antagonists (VKA), which requires frequent monitoring and has numerous drug and dietary interactions. NOAC provides a potent and a predictable therapeutic effect with comparatively minimal routine laboratory monitoring required.

Multiple risk prediction scores have been introduced to assist in the assessment and stratification of bleeding risk. The hypertension, abnormal renal/liver function, stroke, bleeding history or predisposition, labile international normalised ratio, elderly, drugs/ alcohol concomitantly (HAS-BLED) score is among the most commonly used scores for bleeding risk stratification and is used to assess bleeding risk in patients with AF. A patient with a HAS-BLED score [?]3 is considered to be at high-risk for bleeding. ${ }^{13}$ The use of the HASBLED score specifically for patients on concomitant DAPT however has not been validated. The PREdicting bleeding Complications In patients undergoing Stent implantation and subsequent (PRECISE)-DAPT score was introduced to assess bleeding risk for patients on DAPT. A longer duration of DAPT is associated 
with significantly increased bleeding in patients at high PRECISE-DAPT risk (score[?]25). ${ }^{14}$ Data support an ischemic benefit of DAPT only in patients with a PRECISE-DAPT score <25.16) Both bleeding risk prediction models have utility for predicting future significant bleeding events in patients taking an oral anticoagulants (OAC) and undergoing PCI. ${ }^{15}$

An overview of the pivotal NOAC trials in patients with non-valvular AF are summarized in Table 1. In the Randomized Evaluation of Long-term anticoagulant therapy (RE-LY) trial, dabigatran was compared to warfarin in 18,113 patients with results first published in 2009. ${ }^{16}$ In 2011, results were published from the pivotal Rivaroxaban Once Daily Oral Direct Factor Xa Inhibition Compared with Vitamin K Antagonism for Prevention of Stroke and Embolism Trial in AF (ROCKET-AF) trial of 14,246 patients comparing rivaroxaban to warfarin. ${ }^{17}$ The Apixaban for Reduction in Stroke and Other Thromboembolic Events in AF (ARISTOTLE) trial compared apixaban to warfarin and assessed outcomes in 18,201 patients. ${ }^{18}$ Effective Anticoagulation with Factor Xa Next Generation in AF (ENGAGE-AF) compared edoxaban to warfarin in 21,105 patients with results reported in $2013 .{ }^{19}$

As discussed earlier the PIONEER AF-PCI trial and the RE-DUAL trial both compared a NOAC plus single antiplatelet therapy with triple therapy with a VKA plus DAPT. Both pivotal trials reported significantly lower bleeding with the dual antithrombotic regimen compared with triple therapy. ${ }^{20-22}$

In the RE DUAL PCI AF, patients with atrial fibrillation who had undergone PCI, the risk of bleeding was lower among those who received dual therapy with dabigatran and a $\mathrm{P} 2 \mathrm{Y}_{12}$ inhibitor than among those who received triple therapy with warfarin, a $\mathrm{P}_{2} \mathrm{Y}_{12}$ inhibitor, and aspirin. Dual therapy was noninferior to triple therapy with respect to the risk of thromboembolic events. In the trial, dabigatran compared to triple therapy with VKA in 2,725 randomized patients was associated with a significant reduction of bleeding events at both $150 \mathrm{mg}$ and $110 \mathrm{mg}$ twice daily dosing with dabigatran. ${ }^{20,21}$ Due to a concern for potential increased risk for ischemic events with the lower dabigatran dose (110 mg twice daily),150 $\mathrm{mg}$ twice daily dosing with dabigatran is preferred with single antiplatelet therapy.

In the PIONEER AF PCI, participants with atrial fibrillation undergoing PCI with placement of stents, the administration of either low-dose rivaroxaban plus a $\mathrm{P} \mathrm{Y}_{12}$ inhibitor for 12 months or very-low-dose rivaroxaban plus DAPT for 1,6 , or 12 months was associated with a lower rate of clinically significant bleeding than was standard therapy with a vitamin $\mathrm{K}$ antagonist plus DAPT for 1, 6, or 12 months. The three groups had similar efficacy rates, but the clinical efficacy is uncertain as the study was not powered to evaluate thrombotic events ( stent thrombosis, ischemic stroke).

The trial randomized 2,124 patients with nonvalvular AF treated with PCI to receive 1 of 3 treatment strategies of antiplatelet therapy and OAC. The investigators reported that the use of either low- dose rivaroxaban plus a P2Y12 inhibitor for 12 months or very-low-dose rivaroxaban plus DAPT was associated with lower clinically significant bleeding compared with VKA plus DAPT. ${ }^{22}$

The AUGUSTUS trial reported the impact of apixaban with and without aspirin in 4,614 patients with $\mathrm{AF}$ and an acute coronary syndrome or PCI. The use of a P2Y12 inhibitor and apixaban without aspirin resulted in less bleeding and fewer hospitalizations without significant differences in the incidence of ischemic events compared with drug regimens that included a vitamin $\mathrm{K}$ antagonist, aspirin, or both. ${ }^{23}$ Edoxaban is currently being studied as part of a triple therapy regimen in the Edoxaban Treatment Versus Vitamin K Antagonist in Patients With AF Undergoing PCI (ENTRUST-AF) trial.

There are a few highlights regarding NOACs which are important to be considered. The various RCTs data has concluded :

1. Apixaban $5 \mathrm{mg}$ twice a day and Dabigatran $150 \mathrm{mg}$ twice a day are most efficacious in prevention of stroke / systemic embolism. Among the two dabigatran $150 \mathrm{mg}$ has shown most efficacy.

2. Apixaban has shown the most favourable outcomes in major bleeding and intracranial haemorrhage in elderly population, making it the safest drug for this group of population.

3. Apixaban should be preferred for patients with renal impairment . 
4. Apixaban $2.5 \mathrm{mg}$ are considered in patients with any of the two present: age $>80$ years, weight $<60$ $\mathrm{kgs}$ and serum creatinine $>1.5 \mathrm{mg}$

\section{GUIDELINE RECOMMENDATIONS}

The 2016 American College of Cardiology/American Heart Association (ACC/AHA) focused update on duration of DAPT in patients with coronary artery disease guideline recommendations for patients at high bleeding risk treated with PCI supports clopidogrel as the P2Y12 inhibitor of choice in patients on OAC therapy. ${ }^{24}$ The duration of DAPT recommended by the ACC/AHA guidelines is based on the indication for PCI. In patients with stable coronary artery disease, discontinuation of the P2Y12 inhibitor at 3-months is recommended, whereas for patients treated for acute coronary syndrome, P2Y12 inhibitors should be continued for at least 6 months. ${ }^{24}$

The 2018 ESC guidelines similarly recommend clopidogrel as the P2Y12 inhibitor of choice, with low dose ([?]100 mg daily) aspirin, however recommend keeping the duration of triple therapy as short as possible. ${ }^{25}$

ESC guidelines recommend triple therapy with aspirin, clopidogrel and OAC for 1 month, and up to 6 months in patients at higher ischemic risk due to acute coronary syndrome or procedural characteristics that outweigh bleeding risk (class IIa, level of evidence B).

When a NOAC is added to either aspirin or clopidogrel, the lowest approved effective dose for stroke prevention tested in AF trials should be considered (class IIa, level of evidence C).

The ESC cautions that the use of ticagrelor or prasugrel is not recommended as part of triple antithrombotic therapy with aspirin and OAC (class III, level of evidence C).

As per literature and evidence, we noted that majority of the patients were initiated on NOACs by physician's but the duration, dosage and recommendations were varied.

The following are key perspectives from the state-of-the-art review on management of antithrombotic therapy in atrial fibrillation (AF) patients undergoing percutaneous coronary intervention (PCI) published in 2019: 26

1. Most patients with $\mathrm{AF}$ and stroke risk factors require oral anticoagulation (OAC) to decrease their risk of stroke or systemic embolism. This is now best achieved using direct oral anticoagulants (DOACs) due to lower rates of intracranial haemorrhage as compared to vitamin $\mathrm{K}$ antagonists (VKAs; e.g., warfarin).

2. Approximately 5-10\% of patients undergoing PCI have comorbid AF, which complicates antithrombotic therapy decisions. While guidelines recommend dual antiplatelet therapy (DAPT) for patients following PCI, this in combination with OAC therapy places patients at high risk for bleeding complications.

3. Several randomized trials have demonstrated that a regimen of a DOAC plus a single antiplatelet agent (usually clopidogrel) provides better safety than a triple therapy regimen of VKA plus DAPT.

4. For patients undergoing PCI with comorbid AF, strategies such as radial access and a brief anticoagulation washout prior to PCI may be considered to help reduce procedure-related bleeding risk. New-generation drug-eluting stents are also preferable, as they require shorter courses of antiplatelet therapy and are associated with a lower risk of stent thrombosis.

5. For patients undergoing PCI with comorbid AF, use of DOACs is preferred over VKAs. DOACs should be prescribed at stroke prevention doses (e.g., apixaban $5 \mathrm{mg}$ BID) or doses specifically tested in AF plus PCI randomized trials (e.g., rivaroxaban $15 \mathrm{mg}$ daily) when possible. Dose reduction with dabigatran (110 mg BID) may be considered in patients at higher bleeding risk.

6. Use of triple antithrombotic therapy (OAC plus DAPT) should be limited in duration as much as possible. In many cases, this can be limited to the peri-PCI period only. Patients at high thrombotic risk may be considered for up to 1 month.

7. When combining OAC and antiplatelet therapy, use of low-dose aspirin (81-100 mg) is preferred. Use of clopidogrel is also preferred over other P2Y12 inhibitor medications and over aspirin in most patients. 
8. Most patients with AF who undergo PCI can discontinue antiplatelet therapy 12 months following stent placement. They should continue on OAC therapy long-term.

\section{RECOMMENDATIONS:}

Our recommendation based on current evidenced-based medicine, best practices, clinical experience and available pharmacologic therapies are the following :

- The approach to a patient with AF and PCI is summarized in various tables 1,2 and 3 .

- Prognosis is worse in those experiencing an adverse event whether it is an ischemic or bleeding event following PCI, so efforts are needed to balance these competing risks.58)

- DES should be used as the default choice of stent.

- Aspirin therapy should be limited to the minimal duration required post PCI, with the dose restricted to low-dose aspirin

- NOAC's offer significant advantages over VKA including less drug and dietary interactions, less routine monitoring of blood work, and greater time in therapeutic range, thus they should be the drug of choice in patients of $\mathrm{AF}$ and PCI.

- Potent thienopyridine therapy should be used following stent implantation in the absence of routine P2Y12 testing prior to initiating antiplatelet monotherapy.

- Amongst P2Y12 inhibitors prasugrel is not the preferred drug; clopidogrel or ticagrelor may be used with clopidogrel given preference in view of the enormous literature.

- Patients on anticoagulation undergoing PCI should undergo routine assessment with intravascular imaging as the role of high-risk lesion-related features have increased importance prior to determining optimal duration of treatment with DAPT.

- In RE-DUAL trial, it was shown, that without aspirin, the use of $110 \mathrm{mg}$ dabigatran was associated with a numerical increase in death, myocardial infarction, stroke and stent thrombosis. Thus keeping the ischemic and bleeding risks in mind- Dabigatran $110 \mathrm{mg}$ can be used when patients are in triple therapy treatment which can be switched to Dabigatran 150mg when on dual therapy without aspirin.

- Dabigatran $150 \mathrm{mg}$ and Apixaban $5 \mathrm{mg}$ both are significantly efficacious in prevention of ischaemic stroke, with the former more than the latter.

- Apixaban $2.5 \mathrm{mg}$ should be considered in patients of AF with PCI if age $>80$ years, weight $<60 \mathrm{kgs}$ and serum creatinine $>1.5 \mathrm{mg} / \mathrm{dl}$.

- Though studies on rivaroxaban did provide evidence on decrease in bleeding events but efficacy in view of stent thrombosis and ischemic stroke was uncertain.

- Though the choice of NOACs amongst patients with AF and PCI are subjective to the patient and treating physician, evidence does support dabigatran $150 \mathrm{mg}$ and apixaban $5 \mathrm{mg}$ with minimal but significant room for dabigatran $110 \mathrm{mg}$ and apixaban $2.5 \mathrm{mg}$

\section{LEGENDS:}

TABLE 1: Schematic diagram for use of long term antiplatelet and NOAC therapy. NOAC: Novel oral anticoagulant, A : Aspirin, C : Clopidogrel, T : Ticagrelor

TABLE 2: Diagram depicting the approach for new onset AF and ACS. NOAC: Novel oral anticoagulant, A : Aspirin, C : Clopidogrel, T : Ticagrelor. UFH : Unfractionated Heparin. LMWH : Low Molecular Weight Heparin.

TABLE 3: Diagram depicting the approach for patient of ACS and old AF on treatment (NOAC/ VKA). NOAC: Novel oral anticoagulant. VKA: Vitamin K Antagonist. FFP: Fresh Frozen Plasma. PCI: Percutaneous Coronary Intervention.

\section{TABLE 1}

PCI

TABLE 1: Schematic diagram for use of long term antiplatelet and NOAC therapy. NOAC: Novel oral 
anticoagulant, A : Aspirin, C : Clopidogrel, T : Ticagrelor

TABLE 2

TABLE 2: Diagram depicting the approach for new onset AF and ACS. NOAC: Novel oral anticoagulant, A : Aspirin, C : Clopidogrel, T : Ticagrelor. UFH : Unfractionated Heparin. LMWH : Low Molecular Weight Heparin.

\section{TABLE 3}

TABLE 3: Diagram depicting the approach for patient of ACS and old AF on treatment (NOAC/ VKA). NOAC: Novel oral anticoagulant. VKA: Vitamin K Antagonist. FFP: Fresh Frozen Plasma. PCI: Percutaneous Coronary Intervention. 